\title{
Is a 30 kV CFE-SEM in STEM-in-SEM Mode Suitable for Characterizing Real Materials?
}

Nicolas Brodusch and Raynald Gauvin

McGill University, Montreal, Quebec, Canada

Since recently, low voltage transmission electron microscopes (TEM) were developped to reduce the knock-on damage. Indeed, this process, where atoms are ejected from the material due to the interaction with the energetic electron beam, is favored at medium and high primary beam energies [1]. Typically, at 200 or $300 \mathrm{kV}$, a large fraction of materials in TEM and scanning TEM (STEM) are affected by knockon displacement at different levels. Thus, voltages as low as $20 \mathrm{kV}$ were exploited to produce atomic resolution images and EELS characterization but the reported data concerned mainly reference materials and proof of concept experiments [2]. But what about real materials? Can unambiguously low voltage TEM/STEM produce high level characterization results as high energy microscopes do?

We propose to demonstrate how a low accelerating energy scanning electron microscope (SEM) in STEM mode at $30 \mathrm{kV}$ can provide useful data regarding the sample chemistry and microstructure at the nano and even atomic scale. The instrument used was a Hitachi SU-9000EA cold-field emission SEM (CFE-SEM) fitted with a windowless silicon drift detector, an electron energy-loss spectrometer, an on-axis diffraction camera and bright- and dark-field detectors. This microscope has shown unprecedented spatial resolution for a SEM and was the first to provide lattice resolution as low as $0.16 \mathrm{~nm}$ [3]. The on-axis diffraction camera allows to capture convergent beam as well as pseudo-parallel patterns (spot patterns) which are used to collect orientation data from the specimen and maximize the chances to produce lattice images. In addition, the unique low voltage EELS system of the SU9000EA was proved useful for collecting energyloss spectra from thin materials like graphene [4].

Since then, the literature reporting low voltage STEM imaging and spectroscopy results on real materials is very sparse, planting seeds of doubt in potential user's mind in what to really expect of this new technology. Here, we present results obtained from real materials using a $30 \mathrm{kV}$ CFE-SEM in STEM mode. Figure 1 is an example of lattice imaging that was achieved on h-BN where the (01-11) planes of $0.209 \mathrm{~nm}$ were resolved. The procedure to produce such images will be given in details and the sample preparation as well as the materials electron beam sensitivity will be discussed as well. Figure 2 shows how low voltage STEM-EELS can help characterizing the degree of graphitization of biochar specimens. Convergent beam diffraction was applied on such materials but because they are made of a large part of amorphous material, it was very difficult to quantify the state of graphitization of the different samples since the diffraction patterns are a mixture of amorphous and crystalline phases. It is known, however, that the $\mathrm{C} \mathrm{K}$ edge holds a signature of the crystalline structure of the material, i.e. diamond, graphite or amorphous [4]. In this example, lattice imaging was used to characterize the local distribution of the amorphous and graphite structures (Figure 2a) in the material while STEM-EELS proved to be efficient to evaluate quantitatively the fraction of both phases. 

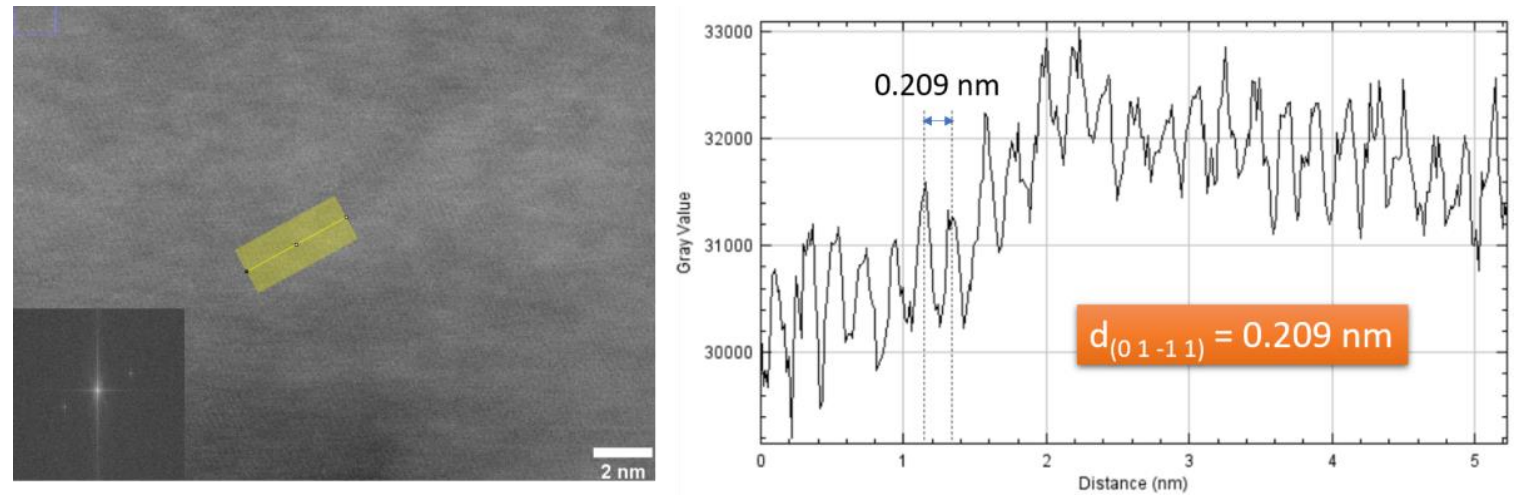

Figure 1. Lattice bright-field image of h-BN with SU9000 CFE-SEM at $30 \mathrm{kV}$ with extracted profile across several lattice planes showing a d-spacing for the (01-11) planes identical to the theoretical one.
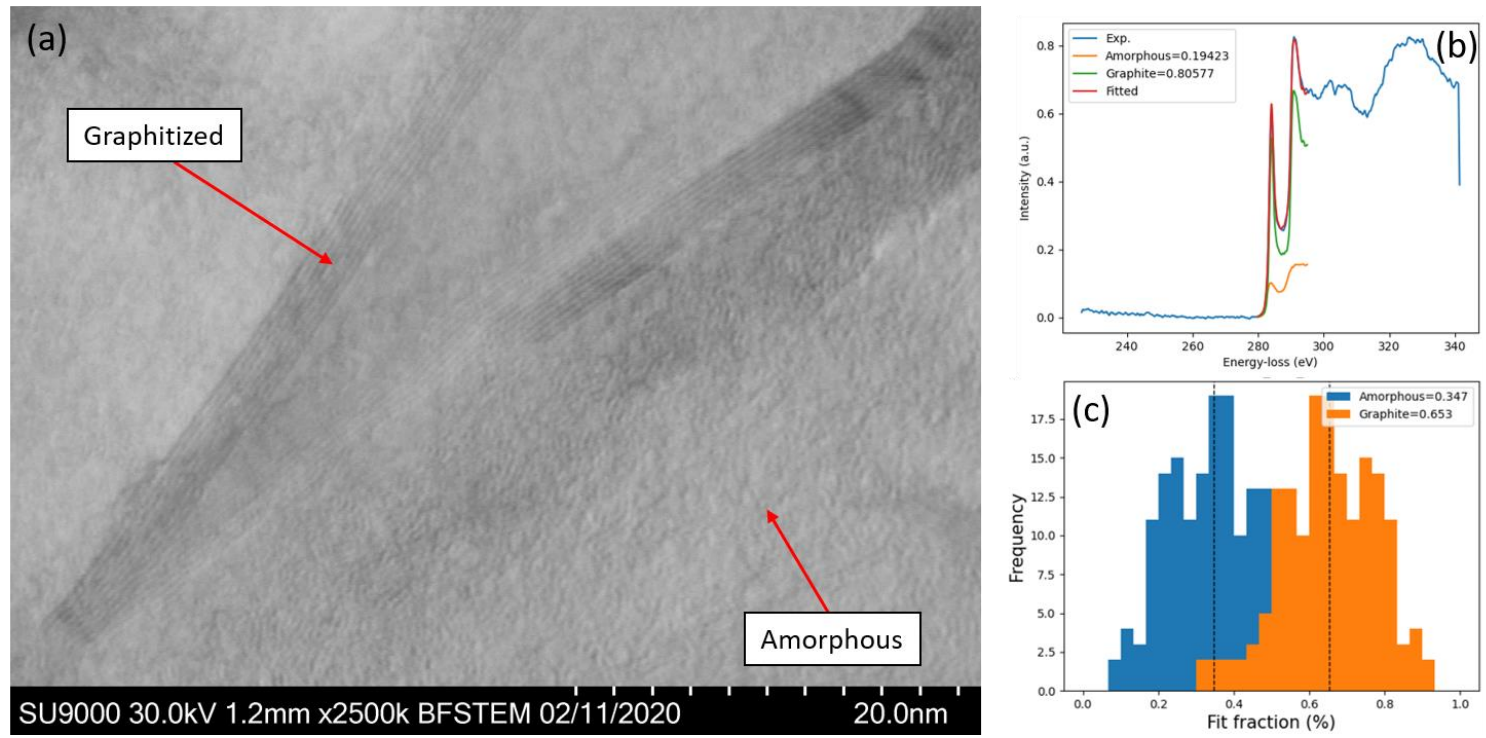

SU9000 30.0kV 1.2mm x2500k BFSTEM 02/11/2020

$20.0 \mathrm{~nm}$

Figure 2. (a) Lattice bright-field image of partially graphitized biochar with SU9000 CFE-SEM at $30 \mathrm{kV}$; (b) experimental EELS spectrum from the same material with fitted profiles from amorphous and graphitic carbon references; (c) amorphous vs graphitic carbon fraction distribution for 164 EELS random measurements.

\section{References}

[1] R.F. Egerton, P. Li, M. Malac, Radiation damage in the TEM and SEM, Micron, 35 (2004) 399-409.

[2] U. Kaiser, J. Biskupek, J.C. Meyer, J. Leschner, L. Lechner, H. Rose, M. Stöger-Pollach, A.N. Khlobystov, P. Hartel, H. Müller, others, Transmission electron microscopy at 20kV for imaging and spectroscopy, Ultramicroscopy, 111 (2011) 1239-1246.

[3] M. Konno, T. Ogashiwa, T. Sunaoshi, Y. Orai, M. Sato, Lattice imaging at an accelerating voltage of $30 \mathrm{kV}$ using an in-lens type cold field-emission scanning electron microscope, Ultramicroscopy, 145 (2014) 28-35.

[4] T. Sunaoshi, K. Kaji, Y. Orai, C.T. Schamp, E. Voelkl, STEM/SEM, Chemical Analysis, Atomic Resolution and Surface Imaging At $\leq 30 \mathrm{kV}$ with No Aberration Correction for Nanomaterials on Graphene Support, Microscopy and Microanalysis, 22 (2016) 604-605. 